\title{
O APOIO DA SUPERINTENDÊNCIA ESCOLAR AOS GESTORES ESCOLARES DO CEARÁ DURANTE A IMPLANTAÇÃO DO ENSINO REMOTO: UMA ANÁLISE SOB A ÓTICA DOS GESTORES DA REGIONAL CREDE 3, ACARAÚ \\ http://dx.doi.org/10.5902/2318133861863
}

\author{
Francisco José da Costa ${ }^{1}$ \\ Nádia Cristina Ribeiro Freitas ${ }^{2}$ \\ Márcia Roberta Silva Azevedo 3
}

\section{Resumo}

Neste artigo tem-se o objetivo de apresentar uma avaliação dos gestores sobre a atuação da Superintendência Escolar da Seduc/CE na orientação, apoio e qualificação da gestão escolar no que diz respeito à implantação e acompanhamento do plano de atendimento domiciliar no período de ensino remoto. Utilizou-se de uma pesquisa qualitativa e exploratória, de natureza aplicada, visando a avaliação do serviço da superintendência pelos gestores e a geração de conhecimentos sobre a prática desse colaborador. A metodologia foi composta por pesquisa bibliográfica, aplicação de questionário estruturado e análise dos resultados contextualizando com a bibliografia. Concluiu-se que o apoio da Superintendência tornou-se essencial no processo de implementação do PAD, uma vez que orientou, acompanhou e gerou reflexão sobre a atuação dos gestores, fortalecendo sua atuação na organização dos processos escolares, com foco na interação, manutenção de vínculo e aprendizagem dos estudantes. Constatou-se também que o serviço da Superintendência gerou uma relação de confiança, corresponsabilização e proximidade das escolas com as demais instâncias da rede, o que contribuiu para uma implantação mais eficaz do plano de atendimento domiciliar das escolas.

Palavras-chave: gestão, superintendência escolar, ensino remoto.

\section{THE SUPPORT OF SCHOOL SUPERINTENDENCE TO SCHOOL MANAGERS DURING THE IMPLEMENTATION OF REMOTE EDUCATION: AN ANALYSIS FROM THE VIEW OF THE REGIONAL BOARD OF EDUCATION IN STATE OF CEARÁ (CREDE 3), ACARAÚ}

\begin{abstract}
$\underline{61863}$
Abstract

This work aims to present an assessment by managers the role of the Superintendence in guiding, supporting and qualifying school management concerning the implementation and monitoring of the Home Care Plan in the period of remote education. A qualitative and exploratory research, of applied nature, was used, aiming at the assessment of the superintendence service by the managers and the generation of knowledge about the practice of this employee. The methodology
\end{abstract}

1 Coordenadoria Regional de francisco.costa0682@gmail.com.

2 Coordenadoria Regional de Desenvolvimento nadiaribeiro25@gmail.com.

3 Coordenadoria Regional de azevedomarcia20@gmail.com.

Regae: Rev. Gest. Aval. Educ. Santa Maria

Desenvolvimento

da Educação

da Educação

da Educação da Seduc/CE, Brasil. E-mail: da Seduc/CE, Brasil. E-mail: da Seduc/CE, Brasil. E-mail: 
consisted of bibliographic research, application of a structured questionnaire, and analysis of the results in context with the bibliography. It was concluded that support of the Superintendence became essential in the process of implementing this plan, since it guides, accompanies, gives feedback, generates reflection on the performance of managers, thus strengthening their performance in the school processes organization, focusing in interaction, maintaining the link and learning from students. It was found that the Superintendence service generates a relationship of trust, co-responsibility and proximity of schools to other instances of the network, which contributed to a more effective implementation of Home Care Plan from the state public schools.

Key-words: management, school superintendence, remote teaching.

\section{Introdução}

m dos maiores desafios das redes de ensino é tornar as políticas públicas eficazes e equitativas. Diante deste desafio e do cenário de pandemia faz-se necessário uma comunicação efetiva e ações articuladas que atinjam, de forma equalizada todas as unidades de ensino, permitindo uma maior qualidade no atendimento aos estudantes, melhoria nos indicadores de aprendizagem, minimizando o risco de um agravamento da desigualdade, diante das desigualdades de acesso dos estudantes. Considera-se que a gestão escolar é um dos principais pilares para assegurar a eficácia do trabalho pedagógico e a melhoria das condições de aprendizagem. Diante de um cenário de desafios da integração da rede e fortalecimento da atuação da gestão escolar, o superintendente escolar tem papel fundamental na implantação e acompanhamento de políticas públicas e no apoio e fortalecimento da liderança da gestão escolar.

De acordo com Paulina (2015) "os supervisores têm um papel estratégico no monitoramento pedagógico para a implantação e a continuidade de políticas públicas, evitando que a rede perca o foco" (p. 8). Nesta perspectiva, a Superintendência tem como função principal manter o gestor centrado no processo pedagógico e na garantia de melhores condições de aprendizagem.

Considerando o cenário desafiante que se instaurou durante o período de pandemia da covid-19, a Superintendência Escolar da Secretaria da Educação do Estado do Ceará, a partir da portaria n. 0268/2020-Gab, de 13 de abril de 2020, instituiu o regime de trabalho especial para os profissionais da educação e implantação imediata de uma política escolar de atendimento aos estudantes durante o isolamento social. Foi elaborado o Plano de Atendimento Domiciliar - PAD - para orientar as atividades da escola com foco em manter a interação com os estudantes, utilizando as diferentes estratégias de ensino remoto. Durante o período de atividades remotas a Superintendência manteve uma comunicação contínua de orientação a gestão sobre rotinas de estudo domiciliar, reflexões individuais e coletivas para melhoria do PAD. Realizou-se ainda, acompanhamento a planejamentos de professores e conselho de turma, bem como visita técnica remota na qual a Superintendência ressignificou os indicadores com foco na manutenção do vínculo da escola com os estudantes, possibilidades de acesso, permanência e aprendizagem, para melhor orientar os gestores diante do desafio atual, de atendimento e garantia da aprendizagem dos estudantes durante o estudo domiciliar. 
O Plano de Atendimento Domiciliar - PAD - é um documento que foi elaborado pelas escolas da rede estadual do Ceará, no qual constam as diretrizes para a organização e sistematização do trabalho escolar durante o período de ensino remoto. No documento estão descritas a organização da escola para os seguintes processos: planejamento das atividades pelos professores; estratégias e ferramentas digitais que serão adotadas pela escola no ensino remoto; estratégias de mobilização e comunicação com os estudantes e famílias; processo de acompanhamento e monitoramento as atividades de estudantes e professores pela gestão escolar; processo de avaliação a ser adotado pela escola. Esse documento foi elaborado a participação de representantes da comunidade escolar e passou a compor o regimento da escola durante o período de ensino remoto, dado o processo de adaptação das escolas ao ensino remoto e a conjuntura de incertezas.

Neste contexto realizou-se uma avaliação dos gestores sobre a atuação da Superintendência Escolar na orientação, apoio e qualificação da atuação da gestão escolar na implantação, acompanhamento e melhoria do plano de atividades domiciliares durante o período de ensino remoto.

A pesquisa foi realizada com gestores de quatro escolas de ensino médio regular, uma escola de ensino médio integrado a educação profissional e uma escola de ensino médio de tempo integral, totalizando um grupo de 18 gestores, considerando diretor escolar e coordenadores pedagógicos. As escolas são acompanhadas pela $3^{\text {a }}$ Coordenadoria Regional de Desenvolvimento da Educação - Crede 3, localizada no município de Acaraú/CE.

\section{O superintendente escolar nas políticas educacionais da rede de ensino do Estado do Ceará.}

Estudos voltados a identificar aspectos positivos nas redes de ensino que apresentam melhoria contínua dos seus indicadores de aprendizagem indicam que esses sistemas de ensino apresentam em comum a gestão escolar como centro do processo. Tais pesquisas também têm identificado a presença de um outro profissional, o supervisor escolar ou superintendente, como é conhecido na rede do Estado do Ceará. A Superintendência Escolar apresenta-se como essencial para a interlocução entre a secretaria e as escolas, apoiando a gestão no alinhamento das ações pedagógicas com foco na aprendizagem:

A Superintendência Escolar foi um órgão decisivo para alinhar as metas e a gestão das escolas à política da Secretaria da Educação e para consolidar, nas unidades escolares, a cultura do monitoramento dos resultados com base nos indicadores. Além disso, pôde apoiar as escolas na compreensão da importância de alguns conteúdos e práticas: ofereceu aos diretores sugestões de organização estética do ambiente escolar. (Brasil, 2005, p. 55)

Em 2008 a Superintendência Escolar foi implantada como uma das ações do Programa Aprender pra Valer, com o objetivo de desenvolver estratégias de acompanhamento da gestão escolar com foco no aperfeiçoamento do trabalho pedagógico e na aprendizagem do aluno. De acordo com Pereira (2010) "a Seduc espera que a ação do Superintendente Escolar gere reflexão e aprendizagem nas escolas, oportunizando ao diretor sair de seu cotidiano para refletir sobre pontos centrais da 
escola" (p. 25). Nesse aspecto, a Superintendência tem um papel importante no acompanhamento e monitoramento do fluxo dos indicadores escolares, integrando na rede as ações estratégicas com foco no acesso, permanência e sucesso dos estudantes no ensino médio.

A partir do acompanhamento sistemático da Superintendência Escolar é possível conhecer as problemáticas vivenciadas no cotidiano escolar, apoiar na reestruturação do projeto político-pedagógico, gerar uma maior autonomia da escola. Ao participar de todos esses processos a Superintendência Escolar desenvolve uma relação de parceria e confiança com a gestão escolar, o que favorece uma maior aceitabilidade e participação da comunidade escolar nas políticas públicas desenvolvidas pela rede de ensino. Dada a importância da Superintendência Escolar para a gestão de políticas públicas da rede estadual do Ceará, esse profissional precisa atender a um perfil adequado para sua atuação, sendo detentor de conhecimentos técnicos e pedagógicos capazes de sensibilizar e mobilizar a gestão escolar para manter o foco na aprendizagem dos estudantes.

\section{A atuação da Superintendência Escolar na gestão escolar para resultados de aprendizagem}

O ensino médio sempre foi desafiante para as redes estaduais de ensino, dada a grande dimensão e diversidades de contextos nas quais as unidades de ensino estão inseridas. Pesquisas apontam que a implantação de uma agenda estratégica focada em ações pedagógicas da rede pressupõe a qualidade da gestão escolar como premissa para se conquistar bons resultados educacionais.

De acordo com Lück (2009) o gestor escolar têm a função de promover na escola o sentido de unidade e garantir padrões elevados de ensino, orientado por diretrizes inclusivos, de equidade e respeito a diversidade, de modo que todos os alunos tenham sucesso escolar e se desenvolvam o mais plenamente possível. Esta posição nos remete a pensar que é preciso garantir apoio formativo ao gestor escolar, de forma que a Superintendência Escolar pode ser esse profissional capaz de garantir de forma contínua apoio técnico e pedagógico, fortalecendo a liderança do gestor frente ao desenvolvimento de uma agenda escolar focada na melhoria dos indicadores de aprendizagem.

De acordo com Freitas (2017) a implantação da Superintendência Escolar pela Seduc/CE pretende "realizar o acompanhamento sistemático às escolas com fins de fortalecer o trabalho do Núcleo Gestor, visando ao desenvolvimento da gestão pedagógica como principal estratégia para alavancar a melhoria dos principais indicadores educacionais e assegurar o sucesso educacional" (p. 16).

Pensar em uma gestão escolar para resultados de aprendizagem é, ao mesmo tempo, considerar a responsabilidade da rede em fortalecer a atuação do gestor escolar e desenvolver um planejamento estratégico com metas claras e um fluxo de comunicação que vise a corresponsabilização de todos em ações focadas na aprendizagem. $O$ Superintende Escolar tem a função de apoiar os gestores na orientação e sistematização dos principais processos e procedimentos da gestão escolar, estimulando-os a manter um olhar reflexivo sobre suas práticas cotidianas, oferecendo subsídios para que possam avançar e aprimorar a qualidade e a eficiência de seu trabalho educativo (Unibanco, 2020). A implementação do circuito de gestão na rede estadual de ensino "permite aos 
gestores escolares a precisão e o rigor necessários para lidar com a complexa realidade escolar, identificando desafios e potencialidades para a construção de melhores caminhos e tomadas de decisões mais acertadas" (Unibanco, 2020, p. 79).

A impacto do circuito de gestão na melhoria da atuação do gestor escolar está diretamente ligado com a atuação da Superintendência Escolar, uma vez que este profissional apoia tecnicamente a gestão na organização do plano de ação e íntegra na rotina da rede uma sistemática de acompanhamento e monitoramento aos indicadores da escola, ao mesmo tempo que capacita os gestores para atuar de forma efetiva na condução da melhoria dos processos de pedagógicos.

Considerando que durante o período de ensino remoto devido à pandemia as orientações da Secretaria de Educação se voltaram para a garantia da interação e manutenção do vínculo com os estudantes, possibilitando-lhes, por meio de atividades remotas, a continuidade dos estudos, evitando-se a reprovação e o abandono escolar, o Conselho Nacional de Educação emitiu o parecer n. 05/2020 orientando as redes de ensino a se readequarem a nova condição. De acordo com o parecer a realização de atividades pedagógicas não presenciais requer readequação, inovação e criatividade, levando-se em consideração o atendimento dos objetivos de aprendizagem e o desenvolvimento das competências e habilidades a serem alcançados pelos estudantes em circunstâncias excepcionais.

Seguindo as orientações da Seduc/CE e de órgãos normativos como o Conselho Nacional de Educação, a Superintendência Escolar atuou na orientação e acompanhamento aos gestores com vistas à qualificação do plano de atendimento domiciliar, aperfeiçoamento do processo avaliativo, considerando aspectos qualitativos e um processo formativo, garantindo assim, diferentes possibilidades de manutenção da aprendizagem no período de distanciamento social.

\section{Resultados e discussão}

Os dados obtidos representam as contribuições de 18 gestores, considerando o público total de coordenadores e diretores. A partir do questionário foram analisadas seis questões relacionadas ao apoio da Superintendência na orientação, acompanhamento e monitoramento do plano de atendimento domiciliar. Após o tratamento dos dados foram obtidos os seguintes resultados de acordo com as questões abordadas a seguir.

$\mathrm{Na}$ questão 1 foi perguntado se durante a organização do plano de atendimento domiciliar o superintendente escolar ofereceu orientação e feedback que contribuiu para o fortalecimento e aperfeiçoamento do plano: 100\% dos gestores responderam afirmativamente. Quando foram analisados os comentários dos gestores que evidenciaram a resposta à questão 1, destacaram-se as seguintes citações:

"O superintendente participou de todas as atividades da escola nesse período, sempre orientando e tirando dúvidas."

"O superintendente foi bem atuante fazendo as devidas correções de rotas e sugerindo estratégias que viessem ao encontro da solução para os problemas vivenciados."

"Esteve presente dando suporte à gestão durante a elaboração do documento (Independente de dia e horário). Estava sempre disponível." 
"O superintendente manteve comunicação diária oferecendo ajuda, dando suporte e sugestões, acompanhando nosso processo de ensino remoto."

"A partir dos contatos diários e da proximidade com o núcleo gestor, o superintendente sempre foi muito propositivo no que diz respeito à construção e reconstrução do instrumental plano de atendimento domiciliar."

"A elaboração das questões motivadoras, o acompanhamento da construção, a orientação para as correções necessárias, contribuíram para elaboração e aprimoramento do nosso plano."

Considerando que os gestores reconhecem 0 apoio e a participação do superintendente escolar no processo de elaboração do PAD e analisando os comentários dos gestores, é possível inferir que a proximidade e assiduidade do superintendente com a gestão possibilitou um maior aprimoramento do plano e apoio à gestão na sua qualificação ao longo da implantação.

$\mathrm{Na}$ questão 2 foi perguntado se a Superintendência Escolar instigou à gestão na busca pelo aperfeiçoamento da mediação pedagógica, bem como na organização de instrumentos de acompanhamento e monitoramento das atividades de atendimento remoto aos estudantes. Nesse aspecto $100 \%$ dos gestores responderam afirmativamente, o que demonstra que o superintendente apoiou a gestão no aperfeiçoamento de sua prática escolar, gerando um circuito efetivo de acompanhamento, monitoramento e ajuste de ações, visando o melhor atendimento aos estudantes durante o período de ensino remoto.

Na questão 3 buscou-se conhecer a análise do gestor sobre a visita técnica que ocorreu de forma remota, na qual os gestores deveriam assinalar os pontos da visita que o superintendente desenvolveu de forma positiva, contribuindo com o fortalecimento da atuação da gestão junto aos processos. A partir da análise das respostas, obteve-se os seguintes resultados:

- $88 \%$ assinalaram que a abordagem na acolhida contextualizou os objetivos da visita e motivou para a condução dos demais pontos da pauta de acompanhamento.

- 88\% indicaram que a apresentação dos objetivos da visita foi bem contextualizando com o cenário e os desafios vivenciados pela escola.

- 100\% consideram que a condução da visita técnica se deu de forma tranquila, na qual a superintendência realizou uma escuta ativa, sendo empática as dificuldades apresentadas pela gestão, trazendo reflexões importantes para a tomada de decisões pela gestão.

- 100\% consideram que durante a visita técnica os indicadores de aprendizagem foram abordados de forma clara, considerando a realidade da escola, proporcionando uma visão geral dos desafios presentes nos processos de ensino, refletindo e mobilizando a gestão para alinhamento e aperfeiçoamento dos processos de intervenção pedagógica.

- 100\% consideram que encaminhamentos da visita técnica foram claros e propositivos para o alinhamento e atuação da gestão frente as intervenções pedagógicas necessárias no período de ensino remoto. 
O resultado da análise que os gestores fazem aos pontos abordados pela Superintendência Escolar durante a visita técnica de acompanhamento remoto evidenciam a boa condução da pauta pela superintendência, destacando-se ainda que a contextualização dos pontos da pauta com a realidade da comunidade escolar, a escuta ativa aos gestores e as reflexões sobre os desafios enfrentados pela escola são pontos essenciais para o fortalecimento de vínculos e a corresponsabilização de todos pelos resultados de aprendizagem da rede.

$\mathrm{Na}$ questão 4 buscou-se verificar se a Superintendência Escolar mantém uma rotina de diálogo com a gestão compartilhando impressões, materiais e instrumentos necessários ao fortalecimento da liderança do diretor/coordenador nos planejamentos e execução das ações estratégicas para o aperfeiçoamento do ensino remoto. $O$ resultado demonstrou que $100 \%$ dos gestores afirmam haver uma rotina permanente de diálogo entre superintendente e gestão com foco no aperfeiçoamento dos processos. A importância desse diálogo é apresentado nos comentários dos gestores, conforme a seguir:

"O que evidencia os diversos diálogos do superintendente com a gestão é o alinhamento e a qualidade do trabalho escolar sempre a luz das diretrizes estaduais e nacionais. Esse diálogo fácil e propositivo potencializa a ação da gestão escolar em busca de um trabalho qualificado."

"O superintendente diariamente, acompanha as ações desenvolvidas pela escola, quer através de mensagens, quer através do diálogo direto. É muito solícito quando precisamos de apoio, disponibilizando material, orientações, bem como servindo de ponte para resolução de assuntos que perpassam nossa gerência."

"Sempre procura saber das dificuldades e diante do exposto apresenta sugestões e contribuições."

"Troca de informações e comunicação via WhatsApp e e-mail cotidianamente. No diálogo constante com a gestão, o superintendente tem oferecido materiais, sugestões de trabalho, diálogos para autoavaliação e espaço para escuta."

"Neste momento de mudanças, onde o ensino remoto é o principal meio de fazermos aprendizagem e de transmissão do conhecimento, seu apoio como Superintendente tem sido muito importante na definição de estratégias e no fortalecimento das ações de acompanhamento."

"O superintendente sempre envia orientações práticas, instrumentais, material de estudo, além de realizar escuta ativa das situações especificas."

"Diariamente acompanha o processo, e sugere melhoramentos, bem como valida os processos."

"Estamos sempre em contato e mantemos um diálogo qualificado acerca de nossas impressões, conquistas, angústias e sempre que necessário e possível, o superintendente oferece materiais para o aperfeiçoamento da gestão." 
"A atuação do superintendente tem agregado bastante aprendizado a dupla gestora para a condução da escola, inclusive neste momento de atividades remotas."

Considerando os registros dos gestores sobre a rotina de diálogo com a superintendência, observa-se uma comunicação contínua, marcada por um diálogo permanente de apoio, orientação, escuta ativa, feedback e proposições de acordo com as necessidades da escola. Destaca-se ainda que esse diálogo potencializa a ação da gestão escolar, mantendo um sentimento institucional de rede, alinhando as orientações educacionais a nível estadual e local, gerando parceria, relação de confiança e corresponsabilização de todos nos processos voltados à melhoria da qualidade dos resultados educacionais. Neste sentido, a superintendência escolar se apresenta como um elo entre Seduc/CE, Crede e escolas.

Outro aspecto importante nesse processo de diálogo entre superintendência e gestão é que fortalece a relação de confiança e gera um sentimento de parceria, reduzindo o peso das cobranças e sensação de culpa.

$\mathrm{Na}$ questão 5 buscou-se saber se a Superintendência Escolar, ao participar de reuniões com professores e conselhos de turma, contribuiu com reflexões que fortalecessem a corresponsabilização da equipe escolar nas ações com foco na equidade e aprendizagem de todos os estudantes. Neste aspecto $100 \%$ dos gestores afirmam que há uma participação e acompanhamento da superintendência aos momentos com professores, fortalecendo reflexões sobre o pensamento de rede em torno da responsabilidade de todos com o foco na aprendizagem. A participação nos momentos promovidos pela escola para acompanhar a execução dos processos escolares é de importante para a coleta de evidências que geram reflexões e encaminhamentos de melhoria junto aos gestores.

$\mathrm{Na}$ questão 6 avaliou-se em uma escala de 0 a 5 a atuação do Superintendente no apoio à gestão escolar para a implantação das ações pedagógicas e organizacionais do plano de atendimento domiciliar no período de ensino remoto. O resultado demonstrou que $95,2 \%$ dos gestores atribuíram o conceito máximo que é 5. As respostas desta questão estão expostas no gráfico a seguir: 
Gráfico 1 -

Avaliação dos gestores sobre a atuação da Superintendência no apoio à gestão escolar.

Avalie a atuação da Superintendência Escolar no Apoio à Gestão Escolar, onde O(zero) = apoia muito pouco e 5 (cinco) $=$ apoia muito .

18 respostas

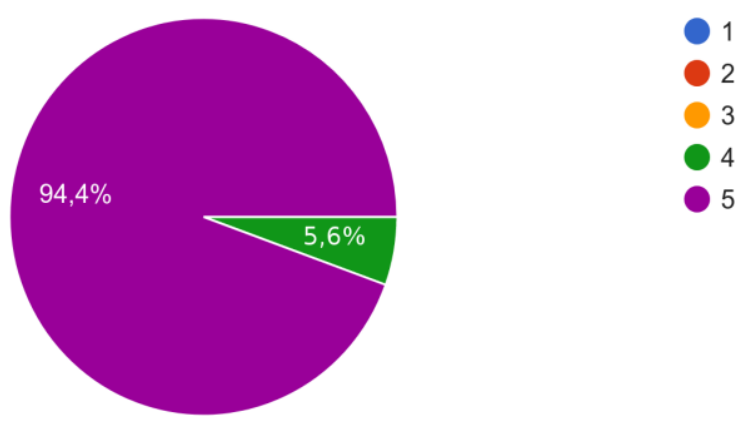

Fonte: autores.

Os dados apresentados no gráfico demonstram que as contribuições e o apoio dado a superintendência é avaliado de forma positiva pelos gestores. Para ilustrar o porquê desse resultado são destacados alguns comentários dos gestores que ratificam a nota atribuída à atuação da Superintendência:

"O superintendente tem sido muito presente e parceiro, contribuindo de forma satisfatória nas nossas ações pedagógicas com zelo profissional."

"Sempre procura saber das dificuldades da escola; apresenta sugestões bastante pertinentes para a dificuldade relatada; promove uma escuta ativa; sempre que possível participa de momentos de planejamento promovidos pela escola; se mostra sempre acessível."

"O superintendente está sempre preocupado com a situação da escola, contribuindo com o processo. É uma pessoa muito ativa, engajada e muito colaborativo."

"Apresenta sugestões de estratégias de intervenção pedagógicos, está sempre disponível para ajudar e esclarecer dúvidas, realizar reflexões sobre o momento e as ações buscando junto com a equipe os melhores caminhos para qualificar o processo de ensino e aprendizagem, é atencioso e orienta tudo de forma bem compreensível e se torna presente no cotidiano da escola."

"Mantém comunicação constante; Demostra interesse no dia a dia da escola; Estuda os dados escolares nas plataformas institucionais; Valoriza o trabalho da escola; incentiva e estimula a gestão no crescimento processual." 
"Diante de todo o cenário em que estamos vivendo, a orientação é muito importante para que a implementação de ações estratégicas sejam objetivas e o trabalho potencializado. Por isso o apoio do Superintendente tem sido muito importante para o fortalecimento de nossa atuação no âmbito escolar."

"Cotidianamente nos atualiza sobre as portarias, decisões, novas estratégias e etc."

As citações feitas pelos gestores para justificarem a nota dada a atuação da superintendência retratam uma parceria positiva que fortalece a gestão escolar, mantendo o foco na melhoria dos processos de aprendizagem. Cabe destacar aspectos importantes para o sucesso da atuação da Superintendência: conhecimento técnico, presença constante em todos os processos, resolutividade, conhecimento dos indicadores da escola, espírito colaborativo, acessibilidade, manutenção de feedback e ser propositivo.

\section{Considerações finais}

Evidenciou-se na pesquisa que a atuação da Superintendência Escolar da Seduc/CE contribui fortemente com a atuação mais assertiva dos gestores em processos desafiantes e urgentes como no caso da implementação do plano de atendimento domiciliar pelas escolas durante o período de pandemia, garantindo a política de atendimento aos estudantes pelo ensino remoto.

De acordo com os resultados obtidos foi possível perceber que a Superintendência Escolar contribuiu para com o fortalecimento da autonomia e liderança dos gestores frente a organização dos processos escolares com foco em resultados de aprendizagem. Esta atuação evidenciou-se durante a implantação do ensino remoto, a partir do importante apoio da Superintendência Escolar aos gestores na organização e execução do plano de atividades domiciliares, fortalecendo o acompanhamento e aperfeiçoamento do papel dos gestores nesse processo.

As análises dos dados demonstraram o reconhecimento pelos gestores da importância da atuação da Superintendência Escolar no suporte técnico, orientação e apoio pedagógico, o que fortalece a atuação dos gestores no processo de organização, acompanhamento e alinhamento das ações da escola no período de implementação do PAD, mantendo-se o foco na interação, manutenção do vínculo, permanência e aprendizagem dos estudantes mesmo no período de isolamento social. Destaca-se, também, a relação de confiança, o diálogo constante entre superintendente e gestão, a presença e acompanhamento as diferentes ações da escola, seguido de feedback de alinhamento aos gestores como princípios essenciais para a boa atuação dos gestores frente aos desafios que surgem no cotidiano escolar.

\section{Referências}

CEARÁ. Superintendência escolar. Fortaleza: Seduc/CE, 2020. Disponível em https://www.seduc.ce.gov.br/superintendencia-escolar/. Acesso em 18 ago. 2020.

CEARÁ. Lei n. 14.190, de 30 de julho de 2008: Programa Aprender Pra Valer. Disponível em https://www.al.ce.gov.br/legislativo/legislacao5/leis2008/14190.htm. Acesso em 18 ago. 2020 . 
CEARÁ. Portaria n. 0268/2020-Gab: institui, em caráter emergencial e temporário, regime especial de trabalho para os servidores e colaboradores da Secretaria da Educação do Estado do Ceará, de 13 de abril de 2020. Disponível em https://www.jusbrasil.com.br/diarios/293135056/doece-13-04-2020-pg-10. Acesso em 18 ago. 2020.

BRASIL. Vencendo o desafio da aprendizagem nas séries inicias: a experiência de Sobral/CE. Brasília: Inep, 200

BRASIL. Parecer n. 05/2020 do Conselho Nacional de Educação: reorganização do calendário escolar em virtude da pandemia do covid 19. Brasília: CNE, 2020. Disponível em

http://portal.mec.gov.br/index.php?option=com docman\&view=download\&alias=145011pcp005-20\&category slug=marco-2020-pdf\&ltemid=30192. Acesso em 10 ago. 2020.

FREITAS, Maria Marlene Vieira. A superintendência escolar na rede pública estadual de ensino do Ceará: perspectivas e desafios. Juiz de Fora: UFJF 2017. 132f. Dissertação (Mestrado em Gestão e Avaliação da Educação Pública). Universidade Federal de Juiz de Fora, Faculdade de Educação.

GIL, Antônio Carlos. Como elaborar projetos de pesquisa. São Paulo: Atlas, 2002.

Iracy Paulina. 10 ações para a rede. Nova Escola, São Paulo, v. 28, 2015. Disponível em https://novaescola.org.br/conteudo/7500/10-acoes-para-a-rede. Acesso em 10 ago. 2020.

INSTITUTO UNIBANCO. Gestão: supervisores são fundamentais no apoio à escola. São Paulo: Instituto Unibanco, 2020. Disponível em https://www.institutounibanco.org.br/aprendizagem-em-foco/55/. Acesso em 10 ago. 2020.

INSTITUTO UNIBANCO. Circuito de gestão: percurso formativo de gestão escolar para resultados de aprendizagem. São Paulo: Instituto Unibanco, 2020.

Lück, Heloisa. Dimensões da gestão escolar e suas competências. Curitiba: Positivo, 2009.

PEREIRA, Antonio Elival. O papel do superintendente escolar como apoio à gestão das escolas de ensino médio no município de Iguatu-Ceará. Juiz de Fora: UFJF 2013. 83f. Dissertação (Mestrado em Gestão e Avaliação da Educação Pública). Universidade Federal de Juiz de Fora, Faculdade de Educação.

Francisco José da Costa é superintendente escolar na 3a Coordenadoria Regional de Desenvolvimento da Educação da Seduc/CE.

Orcid: https://orcid.org/0000-0001-7722-4043.

Endereço: Rua Maria Nilce Couto Cruz, 145 - 62580-000 - Acaraú -CE -Brasil.

E-mail: francisco.costa0682@gmail.com.

Nádia Cristina Ribeiro Freitas é superintendente escolar na 3a Coordenadoria Regional de Desenvolvimento da Educação da Seduc/CE.

Orcid: https://orcid.org/0000-0002-0203-5704.

Endereço: Rua Padre Valderi, 824 - 62595-000 - Cruz - CE- Brasil.

E-mail: nadiaribeiro25@gmail.com. 
Márcia Roberta Silva Azevedo é superintendente escolar na 3a Coordenadoria Regional de Desenvolvimento da Educação da Seduc/CE.

Orcid: https://orcid.org/0000-0001-7085-5360.

Endereço: Rua Coronel Sales, 624 - 62580-000 - Acaraú - CE -Brasil.

E-mail: azevedomarcia20@gmail.com.

Recebido em 14 de agosto de 2020.

Aceito em 21 de janeiro de 2021.

(c) (i) $(\Theta)$ 